\title{
Sex- and age-related variations in the three- dimensional orientations and curvatures of the articular surfaces of the human talus
}

Shuhei Nozaki ( $\sim$ s.nozaki0321@gmail.com )

The University of Tokyo https://orcid.org/0000-0003-2719-1268

Kota Watanabe

Sapporo Medical University

Atsushi Teramoto

Sapporo Medical University

Tomoaki Kamiya

Sapporo Medical University

Masaki Katayose

Sapporo Medical University

Naomichi Ogihara

The University of Tokyo

Research

Keywords: talocrural, subtalar, talonavicular, sex, age, talus

Posted Date: May 19th, 2020

DOl: https://doi.org/10.21203/rs.3.rs-27975/v1

License: (9) (i) This work is licensed under a Creative Commons Attribution 4.0 International License. Read Full License

Version of Record: A version of this preprint was published at Anatomical Science International on November 6th, 2020. See the published version at https://doi.org/10.1007/s12565-020-00585-5. 


\section{Abstract \\ Background}

The high prevalence of foot pathologies in women and the elderly is suggested to be linked to sex-and age-related differences in the calcaneal morphology. Given that, it is expected that there also exists sexand age-related differences of the articular morphology in the keystone of the foot, the talus. The present study investigated the orientation and curvature of the three articular surfaces of the talus in relation to sex and age based on computer tomography (CT).

\section{Methods}

The CT images of 56 feet were used in this study. The orientations of the talocrural, subtalar, and talonavicular joints were quantified three-dimensionally by calculating normal and principal axes of the articular surfaces defined by planar approximation. The curvature radii of the articular surfaces were also quantified by cylindrical and spherical approximations.

\section{Results}

The talonavicular surface was significantly more twisted and less internally rotated in females than in males. With aging, the subtalar articular surface was significantly facing more posteriorly. Moreover, it was found that the curvature radii of the trochlea and navicular articular surfaces significantly increased with aging, indicating the flattening of the articular surfaces.

\section{Conclusion}

The sex- and age-related differences in talar articular morphology were observed. The substantial degeneration pattern of the talar morphology with aging could potentially lead to a higher prevalence of foot disorders in the elderly.

\section{Background}

The talus is articulated superiorly with the tibia and fibula at the talocrural joint, inferiorly with the calcaneus at the subtalar joint, and anteriorly with the navicular at the talonavicular joint. Thus, the talus acts as a mechanical linkage between the foot and the leg, and the orientations of the articular surfaces of the talus determine relative positions and movements of the calcaneus and navicular with respect to the tibia during bipedal locomotion [1-8].

It is well-accepted that the prevalence of the hallux valgus and flatfoot deformity is higher in women [914] and increases with age [13]. While the mechanism underlying these foot disorders remains unclear, 
recent studies suggested that sex- and age-related differences in the calcaneus morphology is possibly linked to higher prevalence of foot disorders in women and elderly $[15,16]$. If this is true, it is expected that there also exists sex- and age-related differences of the articular morphology of the talus, which is the keystone of the foot.

Sex differences in the navicular articular surface of the talus have been reported by Ferrari et al. [17], who indicated that the female navicular articular surface of the talus was more twisted in the frontal plane and more curved than that in males. In contrast, a recent geometric morphometric studies [18-20] has not detected sex differences in the articular surface orientation of the talus. Hence, based on these previous studies, the sex- and age-related differences in the articular morphology of the talus are still under debate. The present study aimed to compare the orientations and curvatures of the articular surfaces of the human talus between males and females, and to explore the correlations between the talar articular morphology and age using three-dimensional computed tomography (CT) images. To extend our knowledge of sex-and age-related differences in the articular morphology of the talus is essential to explain the mechanism underlying the foot disorders, which are more prevalent in women and the elderly.

\section{Methods}

\section{Population}

The tali of the Japanese subjects in a previous analysis of the calcaneus morphology [15] were analyzed in the present study. Briefly, CT scans of subjects without talar and calcaneal injuries or disorders were retrospectively identified in the database at our institution. Fifty-six feet were enrolled in this study (men, 31 feet; women, 25 feet); mean age of men was 49.2 [SD 17.5] years (range, 20-82 years) and that of women was 52.6 [SD 21.6] years (range, 17-87 years). The institutional review board and appropriate ethics committee approved this retrospective review. CT images were acquired using an Aquilion Multidetector CT scanner (Toshiba Medical Systems, Otawara, Japan). Detailed information of the CT image parameters was described in a previous study [15]. The images were imported into a commercial software (Mimics Version 9.0; Materialise Inc., Leuven, Belgium) and a 3-D bone surface of the talus was generated. Mirror image models of left-side specimens were subsequently created using the Geomagic Design X (3-D Systems Inc., Rock Hill, SC, USA) and were treated as right-side specimens.

\section{Articular Surface Analysis}

To quantify the orientations of the articular surfaces, a body-fixed coordinate system was defined for the talus. For this, we used a typical human talus as a standard specimen and defined a body-fixed orthogonal coordinate system with the $X-, Y$-, and $Z$-axes the representing mediolateral, anteroposterior, and dorsoplantar axes, respectively, (Fig. 1) as in a previous report [6]. The origin of the coordinate system was defined as the centroid of the 37 landmarks on the surface of the talus [21]. All specimens 
were superimposed on this standard specimen based on the 37 landmark coordinates [21] using the Generalized Procrustes Analysis method [22-24] to place them in the same coordinate system.

Three regions of the articular surfaces in the talus, i.e., the superior surface of the trochlea, calcaneal articular surfaces, and navicular articular surface, were manually extracted by outlining the visible borders of the corresponding articular surfaces. To quantify the orientation of the three articular surfaces, the planes were fitted to each articular surface using the least-squares method, and the normal vector of each plane was calculated (Fig. 2). Furthermore, the first principal axis was calculated (Fig. 2).

For the trochlea and subtalar articular surfaces (Fig. 2a, b), the angle between the normal vector and the $Y$-axis projected on the sagittal ( $Y Z$-) plane was defined as the superoinferior angle, whereas the angle between the normal vector and the $Z$-axis projected on the frontal ( $X Z$-) plane was defined as the mediolateral angle. The angle between the principal axis and the $Y$-axis projected on the horizontal $(X Y$ - $)$ plane was defined as the rotational angle. For the navicular surface (Fig. 2c), the angles between the normal vector and the $Y$-axis projected on the sagittal plane, between the normal vector and the $Y$-axis projected on the horizontal plane, and between the principal axis and the $Z$-axis projected on the frontal plane were defined as the superoinferior, mediolateral, and rotational angles, respectively. The angles were positive if the surfaces were oriented superiorly, laterally, and internally rotating directions, respectively.

To assess the curvatures of the articular surfaces, the superior trochlea, the posterior calcaneal articular surface, and the navicular surface were approximated by cylindrical and spherical surfaces, using the least-squares minimization (Solid Primitive command in Geomagic Design X, 3D Systems, Rock Hill, SC, USA) (Fig. 3). The subtalar articular facet consisting of the anterior, middle, and posterior calcaneal articular surfaces is essentially a plane joint on which the calcaneus slightly translates and rotates [25]. However, the posterior calcaneal surface is cylindrical and thus approximated by a cylinder. The calculated radii of curvature were normalized by the talar length, defined as the distance between the most distomedial point on the posterior calcaneal articular surface and the center of the navicular articular surface (landmark points of 23 and 36 described in Nozaki et. al. [21]) for comparisons.

\section{Measurement Reproducibility Assessment}

The intra-rater reproducibility for the measurements in each of the 56 tali (two independent measurements per talus) was assessed using the intraclass correlation coefficient with one-way random effects, absolute agreement, single rater/single measurement (ICC, model 1, 1), and 95\% confidence intervals. The standard error of measurement (SEM) was calculated to represent the consistency of the results within individuals in the same unit as the original measurement. The $95 \%$ minimal detectable change $\left(\mathrm{MDC}_{95}\right)$ was also determined for assuming the amount of change required to represent a true change (i.e., exceeding measurement error) [26]. Repeated measurements were conducted by one observer with a 4-week interval. Calculation of the ICC was performed using the SPSS statistics (version 25.0, IBM, Armonk, NY, USA). 


\section{Statistical Analyses}

Multivariate analysis of variance (MANOVA) was individually conducted for each three articular surfaces to investigate sex-related differences in the orientation angles and curvatures as well. Age-related differences in the orientation angles and curvatures of the articular surfaces were also analyzed using the Pearson's correlation coefficient. The statistical significance level was set at $P<0.05$. All statistical analyses were performed in the open source R software, version 3.5.2 (R Core Team, 2016).

\section{Results}

The value of ICC, model 1, 1 for reproducibility of repeated measurements exceeded 0.94 , and $\mathrm{MDC}_{95}$ was less than $2.3^{\circ}$ and $0.8 \mathrm{~mm}$ for the orientation angles and curvature radii, respectively (Table 1 ).

Table 1

Intra-rater reproducibility of repeated measurements

\begin{tabular}{|lllll|}
\hline & ICC & $95 \%$ Cls & SEM & MDC $_{95}$ \\
\hline Superior trochlea surface & & & & \\
\hline Superoinferior angle & 0.980 & $0.966-0.988$ & 0.4 & 1.1 \\
\hline Mediolateral angle & 0.984 & $0.972-0.990$ & 0.3 & 1.0 \\
\hline Rotational angle & 0.985 & $0.975-0.991$ & 0.8 & 2.3 \\
\hline Radius of curvature & 0.990 & $0.998-0.999$ & 0.1 & 0.2 \\
\hline Calcaneal articular surfaces & & & & \\
\hline Superoinferior angle & 0.983 & $0.971-0.990$ & 0.2 & 0.7 \\
\hline Mediolateral angle & 0.940 & $0.899-0.964$ & 0.5 & 1.3 \\
\hline Rotational angle & 0.990 & $0.983-0.994$ & 0.6 & 1.7 \\
\hline Posterior calcaneal articular surface & & & & \\
\hline Radius of curvature & 0.980 & $0.967-0.988$ & 0.3 & 0.8 \\
\hline Navicular articular surface & & & & \\
\hline Superoinferior angle & 0.978 & $0.963-0.987$ & 0.5 & 1.4 \\
\hline Mediolateral angle & 0.989 & $0.982-0.994$ & 0.5 & 1.3 \\
\hline Rotational angle & 0.994 & $0.990-0.997$ & 0.6 & 1.6 \\
\hline Radius of curvature & 0.996 & $0.993-0.998$ & 0.1 & 0.2 \\
\hline $\begin{array}{l}\text { ICC, intraclass correlation coefficient; Cls, confidence intervals; SEM, standard error of measurement; } \\
\text { MDC } 95 \text {, 95\% minimal detectable change }\end{array}$ & & & \\
\hline
\end{tabular}


MANOVA did not detect statistically significant sex differences in the orientations at the superior trochlea and calcaneal articular surfaces, but there was statistically significant overall difference in the orientation angles at the navicular articular surface (Wilks' lambda $=0.846, F=3.16, P=0.03$ ). Particularly, the navicular articular surface in the female talus exhibited significantly lower mediolateral angle (mean differences of $2.5^{\circ}, P=0.03$ ) and lower rotational angle (mean differences of $5.2^{\circ}, P<0.01$ ) compared with that of males (Table 2). There were no significant sex differences in the curvatures of the three articular surfaces (Table 3).

Table 2

Orientation of the articular surfaces of the talus in females and males

\begin{tabular}{|c|c|c|c|c|c|}
\hline & \multicolumn{2}{|c|}{ Females } & \multicolumn{2}{|l|}{ Males } & \multirow[t]{2}{*}{$P$ value } \\
\hline & Mean & $S D$ & Mean & $S D$ & \\
\hline \multicolumn{6}{|c|}{ Superior trochlea surface } \\
\hline Superoinferior angle & 82.0 & 2.9 & 82.7 & 3.0 & 0.4169 \\
\hline Mediolateral angle & -1.1 & 2.6 & -0.4 & 2.8 & 0.3627 \\
\hline Rotational angle & -11.3 & 4.5 & -11.2 & 8.0 & 0.9603 \\
\hline \multicolumn{6}{|c|}{ Calcaneal articular surfaces } \\
\hline Superoinferior angle & -124.2 & 1.7 & -124.3 & 2.0 & 0.7437 \\
\hline Mediolateral angle & 178.6 & 2.0 & 178.9 & 1.6 & 0.4709 \\
\hline Rotational angle & 31.8 & 6.5 & 31.3 & 5.9 & 0.7746 \\
\hline \multicolumn{6}{|c|}{ Navicular articular surface } \\
\hline Superoinferior angle & -33.5 & 3.5 & -34.5 & 3.5 & 0.2716 \\
\hline Mediolateral angle & 12.1 & 4.9 & 14.6 & 3.5 & $0.0272^{*}$ \\
\hline Rotational angle & 68.2 & 8.8 & 73.4 & 5.1 & $0.0085^{\star}$ \\
\hline
\end{tabular}


Table 3

Size-normalized curvatures of the articular surfaces of the talus in females and males

\begin{tabular}{|llllll|}
\hline & \multicolumn{2}{c}{ Females } & & Males & \multirow{2}{*}{ Pvalue } \\
\cline { 2 - 5 } & Mean & SD & Mean & SD & \\
\hline Superior trochlea surface & 2.56 & 0.17 & 2.57 & 0.20 & 0.8317 \\
\hline Posterior calcaneal articular surface & 2.92 & 0.31 & 2.96 & 0.26 & 0.6641 \\
\hline Navicular articular surface & 2.93 & 0.21 & 3.01 & 0.17 & 0.1606 \\
\hline *Significant differences between females and males & & & \\
\hline
\end{tabular}

There was a significant negative correlation between the superoinferior angle of the calcaneal articular surfaces and age $(R=-0.357, P<0.01$, Fig. $4 a)$, indicating that the articular surface is more posteriorly oriented with increasing age. However, there were no significant age-related differences in the other orientation angles. The curvatures of the superior trochlea and navicular articular surface were significantly correlated with age $(R=-0.436, P<0.001 ; R=-0.476, P<0.001$, respectively) (Fig. 4b), but the significant age-related difference of the curvature of the posterior calcaneal surface was not detected.

\section{Discussion}

The present study demonstrated that the navicular surface of the talus was significantly more twisted and less internally oriented in females than in males, as previously suggested $[17,21]$ although such sexassociated differences in the orientations of the articular surfaces were not observed in the superior trochlea and calcaneal articular surfaces. The biomechanical effect of the greater torsion angle in the female talus is obscure, but a recent study by Louie et al. [27] indicated that the navicular articular surface significantly faces to more inferior direction in the sagittal plane and exhibits greater torsion angle in patients with flatfoot. Thus, the greater torsion angle of the navicular surface of the talus may predispose women to developing flatfoot. Further studies are certainly needed to investigate the effect of the orientation of the navicular articular surface of the talus on the pathogenetic mechanism of the flatfoot deformity.

The present study demonstrated that there are considerable age-associated changes in the articular surface morphology of the talus. Firstly, the calcaneal articular surface was found to be significantly more posteriorly oriented with increasing age. If the articular surface is more posteriorly oriented, it means that the surface is more steeply sloping in the anteroposterior direction. Recent morphological study by Nozaki et al. [16] reported that the talar articular surface of the female calcaneus flattens with aging. Therefore, the calcaneus should easily move in the superoposterior direction with respect to the talus during weight-bearing, possibly leading to the larger excessive mobility of the subtalar joint with increasing age. It is suggested that the excessive mobility of the subtalar joint during weight-bearing is linked to foot disorders such as flatfoot deformity $[28,29]$. Several studies actually demonstrated that the 
medial longitudinal arch tends to lower and flatten with aging [30-32]. Consequently, the change in the orientation of the calcaneal articular surface with aging could be a morphological factor for the higher prevalence of flatfoot with aging [30-32].

The present study also revealed that the curvatures of the superior talar trochlea and navicular articular surfaces significantly decreased with age, indicating that the articular surfaces flattened as the age increased. This is possibly due to joint surface remodeling caused by loading accumulation over a long period of time. In fact, a large compressive force is applied to the talocrural joint in a weight-bearing situation $[33,34]$. Therefore, such accumulation of the large compressive force applied to the superior trochlea and navicular articular surfaces over the years may cause talus remodeling. This result is consistent with the fact that the trochlea is generally flattened particularly in the medial border in ankle osteoarthritis patients [35].

There are some limitations associated with the present study. First, this was a cross-sectional study, thus we could not continually follow up the age-related changes in the articular surface morphology of the talus in the same participants. Second, the feet used in this study were not from purely healthy subjects, but from those who presented acute foot or ankle symptoms. Therefore, we strictly set the exclusion criterion to eliminate any foot disorders [15]. Third, the borders of the articular surfaces were manually extracted and might influence the generalizability of our findings. However, the intra-rater reproducibility of all measurements was good; hence, this manual extraction did not affect the main conclusion of this study.

\section{Conclusion}

The sex-related differences of the articular morphology of the talus were found only at the navicular articular surface. The calcaneal articular surfaces significantly oriented more posteriorly with aging, which could consequently cause posterior sliding motion of the calcaneus respective to the talus, possibly leading to the medial longitudinal arch flattening. Furthermore, the superior trochlea and the navicular articular surfaces significantly flattened with aging. In the future, biomechanical studies will be needed to clarify the effect of the sex-and age-related morphological variations in the articular surfaces of the talus on the kinematics of the talocrural, subtalar, and talonavicular joints for better understanding of the pathogenesis of foot disorders, which are prevalent in women and elderly.

\section{Declarations}

\section{Acknowledgements}

The authors express their sincere gratitude to the members of Department of Orthopedic Surgery and Imaging Diagnosis at the Hokkaido Obihiro Kyokai Hospital for providing us with the computer tomography images.

\section{Author's contributions}


SN, MK and NO conceived the study and KW, AT and TK managed the collection of the computed tomography images. SN analyzed data and KW, AT, TK, MK and NO interpreted the results. SN drafted the manuscript and all authors provided critical revision and approved the final version of the article.

\section{Funding}

This study was financially supported by a Grant-in-Aid from the Japanese Society for the Promotion of Science for Research Fellow (grant no. 19J00277).

\section{Availability of data and materials}

The datasets used and analyzed in this study are available from the corresponding author on reasonable request.

\section{Ethics approval and consent to participate}

The retrospective protocols were approved by the Sapporo Medical University Ethics Committee (\#29-246) and the institutional review board in Obihiro Kyokai Hospital (\#2017-12).

\section{Consent for publication}

Not applicable

\section{Conflicts of interest}

The authors have no conflict of interest with regard to this work.

\section{References}

1. Day MH, Wood BA. Functional affinities of the Olduvai Hominid 8 talus. Man. 1968;3:440-55.

2. Latimer B, Ohman JC, Lovejoy CO. Talocrural joint in African hominoids: implications for Australopithecus afarensis. Am J Phys Anthropol. 1987;74:155-75.

3. Gebo DL. Plantigrady and foot adaptation in African apes: implications for hominid origins. Am J Phys Anthropol. 1992;89:29-58.

4. Kidd RS, Ohiggins $\mathrm{P}, \mathrm{Oxnard} \mathrm{CE}$. The $\mathrm{OH} 8$ foot: a reappraisal of the functional morphology of the hindfoot utilizing a multivariate analysis. J Hum Evol. 1996;31:269-91.

5. DeSilva JM. Functional morphology of the ankle and the likelihood of climbing in early hominins. Proc Natl Acad Sci U S A. 2009;106:6567-72.

6. Kanamoto S, Ogihara N, Nakatsukasa M. Three-dimensional orientations of talar articular surfaces in humans and great apes. Primates. 2011;52:61-8.

7. Prang TC. The subtalar joint complex of Australopithecus sediba. J Hum Evol. 2016;90:105-19. 
8. Sorrentino R, Carlson KJ, Bortolini E, Minghetti C, Feletti F, Fiorenza L, et al. Morphometric analysis of the hominin talus: Evolutionary and functional implications. J Hum Evol. 2020;142:102747.

9. Holmes GB Jr, Mann RA. Possible epidemiological factors associated with rupture of the posterior tibial tendon. Foot Ankle. 1992;13:70-9.

10. Coughlin MJ, Jones CP. Hallux valgus: Demographics, etiology, and radiographic assessment. Foot Ankle Int. 2007;28:759-77.

11. Cho NH, Kim S, Kwon DJ, Kim HA. The prevalence of hallux valgus and its association with foot pain and function in a rural Korean community. J Bone Joint Surg Br. 2009;91:494-8.

12. Nguyen US, Hillstrom HJ, Li W, Dufour AB, Kiel DP, Procter-Gray E, et al. Factors associated with hallux valgus in a population-based study of older women and men: The MOBILIZE Boston Study. Osteoarthritis Cartilage. 2010;18:41-6.

13. Nix S, Smith M, Vicenzino B. Prevalence of hallux valgus in the general population: A systematic review and meta-analysis. J Foot Ankle Res. 2010;3:21.

14. Dufour AB, Casey VA, Golightly YM, Hannan MT. Characteristics associated with hallux valgus in a population-based foot study of older adults. Arthritis Care Res. 2014;66:1880-6.

15. Nozaki S, Watanabe K, Kamiya T, Katayose M, Ogihara N. Three-dimensional morphological variations of the human calcaneus investigated using geometric morphometrics. Clin Anat. 2019.

16. Nozaki S, Watanabe K, Kamiya T, Katayose M, Ogihara N. Sex- and age-related morphological variations in the talar articular surfaces of the calcaneus. Ann Anat. 2020;229:151468.

17. Ferrari J, Hopkinson DA, Linney AD. Size and shape differences between male and female foot bones: Is the female foot predisposed to hallux abducto valgus deformity? J Am Podiatr Med Assoc. 2004;94:434-52.

18. Moore ES, Kindig MW, McKearney DA, Telfer S, Sangeorzan BJ, Ledoux WR. Hind- and midfoot bone morphology varies with foot type and sex. J Orthop Res. 2019;73:744-59.

19. Tumer N, Arbabi V, Gielis WP, de Jong PA, Weinans H, Tuijthof GJM, et al. Three-dimensional analysis of shape variations and symmetry of the fibula, tibia, calcaneus and talus. J Anat. 2019;234:132-44.

20. Sorrentino R, Belcastro MG, Figus C, Stephens NB, Turley K, Harcourt-Smith W, et al. Exploring sexual dimorphism of the modern human talus through geometric morphometric methods. PloS one. 2020;15.

21. Nozaki S, Watanabe K, Kamiya T, Katayose M, Ogihara N. Morphological variations of the human talus investigated using three-dimensional geometric morphometrics. Clin Anat. 2020.

22. Gower JC. Generalised Procrustes analysis. Psychometrika. 1975;40:33-50.

23. Rohlf FJ, Slice D. Extensions of the Procrustes method for the optimal superimposition of landmarks. Syst Zool. 1990;39:40-59.

24. Goodall C. Procrustes methods and the statistical analysis of shape. J R Stat Soc Series B. 1991;53:285-339. 
25. Kleipool RP, Dahmen J, Vuurberg G, Oostra RJ, Blankevoort L, Knupp M, et al. Study on the threedimensional orientation of the posterior facet of the subtalar joint using simulated weight-bearing CT. J Orthop Res. 2018;37:197-204.

26. Vincent WJ, Weir JP: Statistics in Kinesiology, Fourth Edition edn. Champaign, IL: Human Kinetics; 1994.

27. Louie PK, Sangeorzan BJ, Fassbind MJ, Ledoux WR. Talonavicular joint coverage and bone morphology between different foot types. J Orthop Res. 2014;32:958-66.

28. Kido M, Ikoma K, Imai K, Maki M, Takatori R, Tokunaga $D$, et al. Load response of the tarsal bones in patients with flatfoot deformity: In vivo 3D study. Foot Ankle Int. 2011;32:1017-22.

29. Kido M, Ikoma K, Imai K, Tokunaga D, Inoue N, Kubo T. Load response of the medial longitudinal arch in patients with flatfoot deformity: In vivo 3D study. Clin Biomech. 2013;28:568-73.

30. Staheli LT, Chew DE, Corbett M. The longitudinal arch. A survey of eight hundred and eighty-two feet in normal children and adults. J Bone Joint Surg Am. 1987;69:426-8.

31. Scott G, Menz HB, Newcombe L. Age-related differences in foot structure and function. Gait Posture. 2007;26:68-75.

32. Redmond AC, Crane YZ, Menz HB. Normative values for the Foot Posture Index. J Foot Ankle Res. 2008;1:6.

33. Manter JT. Distribution of compression forces in the joints of the human foot. Anat Rec. 1946;96:313-21.

34. Kimizuka M, Kurosawa H, Fukubayashi T. Load-bearing pattern of the ankle joint. Contact area and pressure distribution. Arch Orthop Trauma Surg. 1980;96:45-9.

35. Seki H, Ogihara N, Kokubo T, Suda Y, Ishii K, Nagura T. Visualization and quantification of the degenerative pattern of the talus in unilateral varus ankle osteoarthritis. Sci Rep. 2019;9:17438.

\section{Figures}




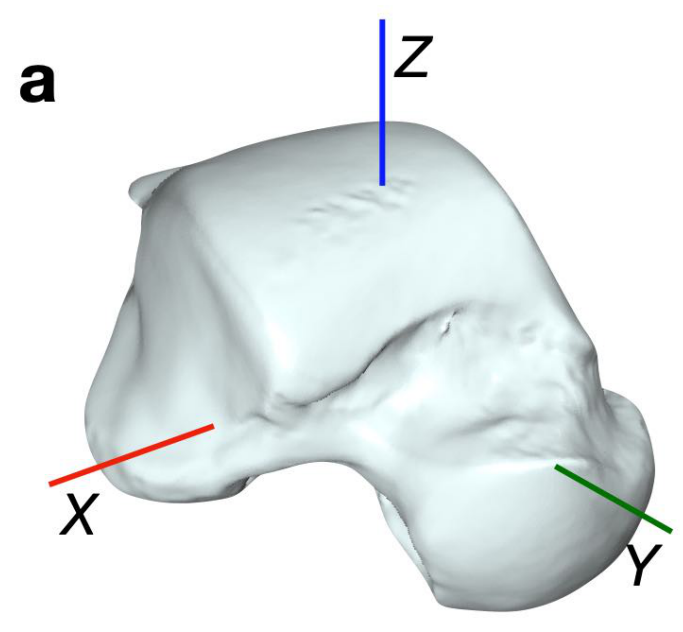

b
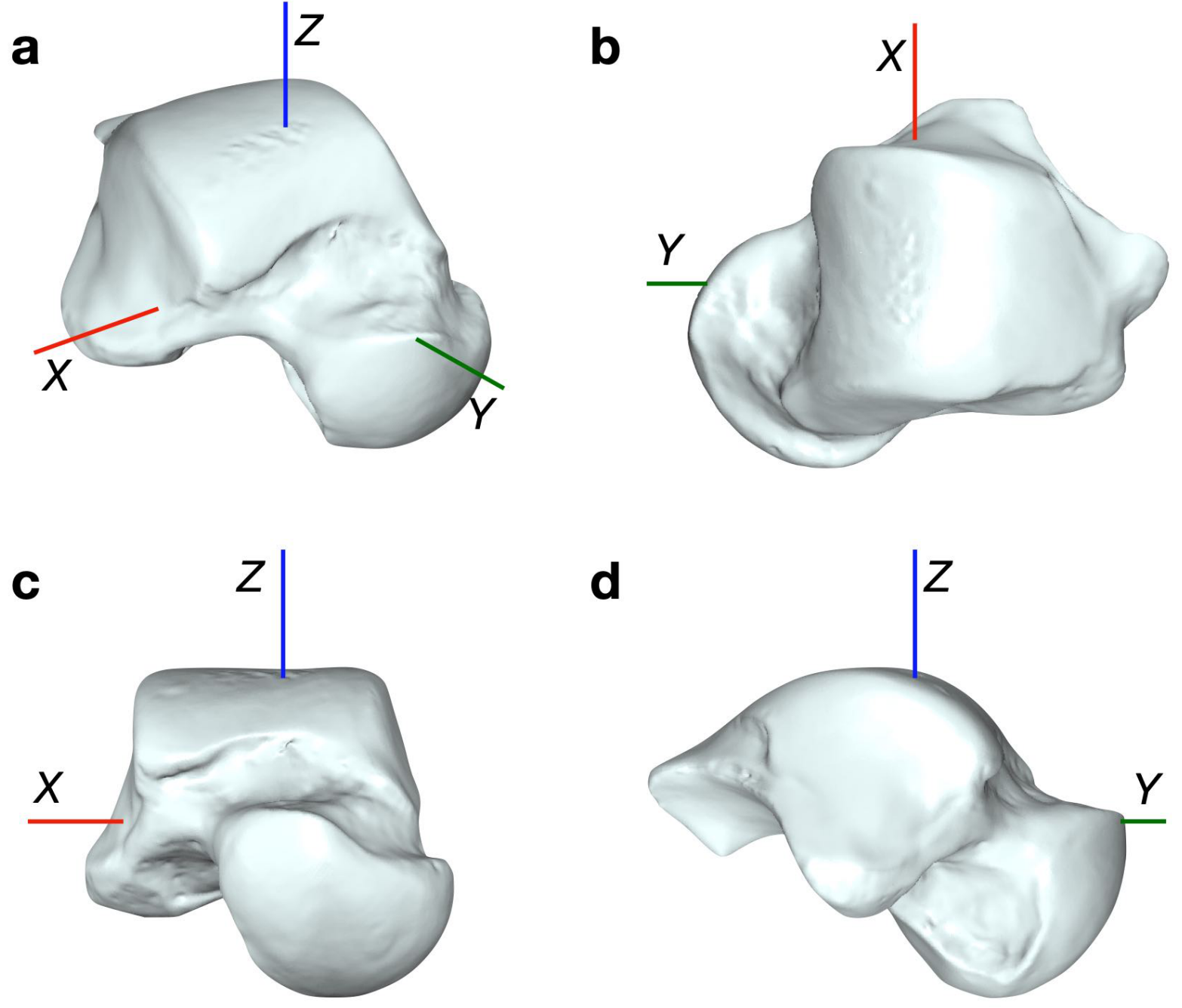

Figure 1

Body-fixed coordinate systems of the talus. Anterolateral (a), superior (b), anterior (c), and lateral (d) views 


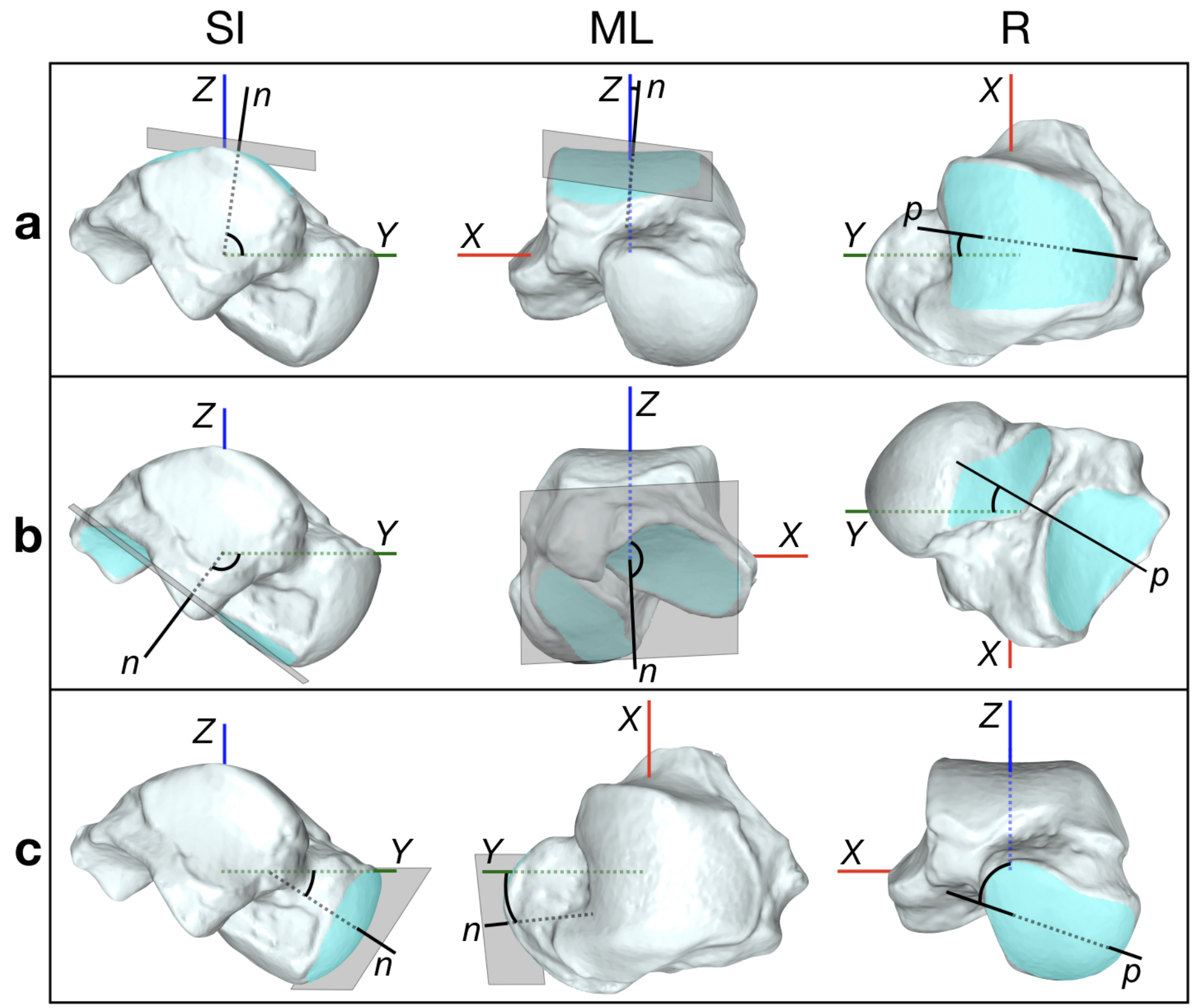

Figure 2

The orientation angles of the articular surfaces of the talus. The planes are fitted to the superior trochlea (a), calcaneal articular surfaces (b), and navicular articular surface (c) and the normal vectors (n) are calculated. The first principal axes ( $p$ ) are also determined on the fitted planes of the three articular surfaces. SI: superoinferior angle, ML: mediolateral angle, R: rotational angle 

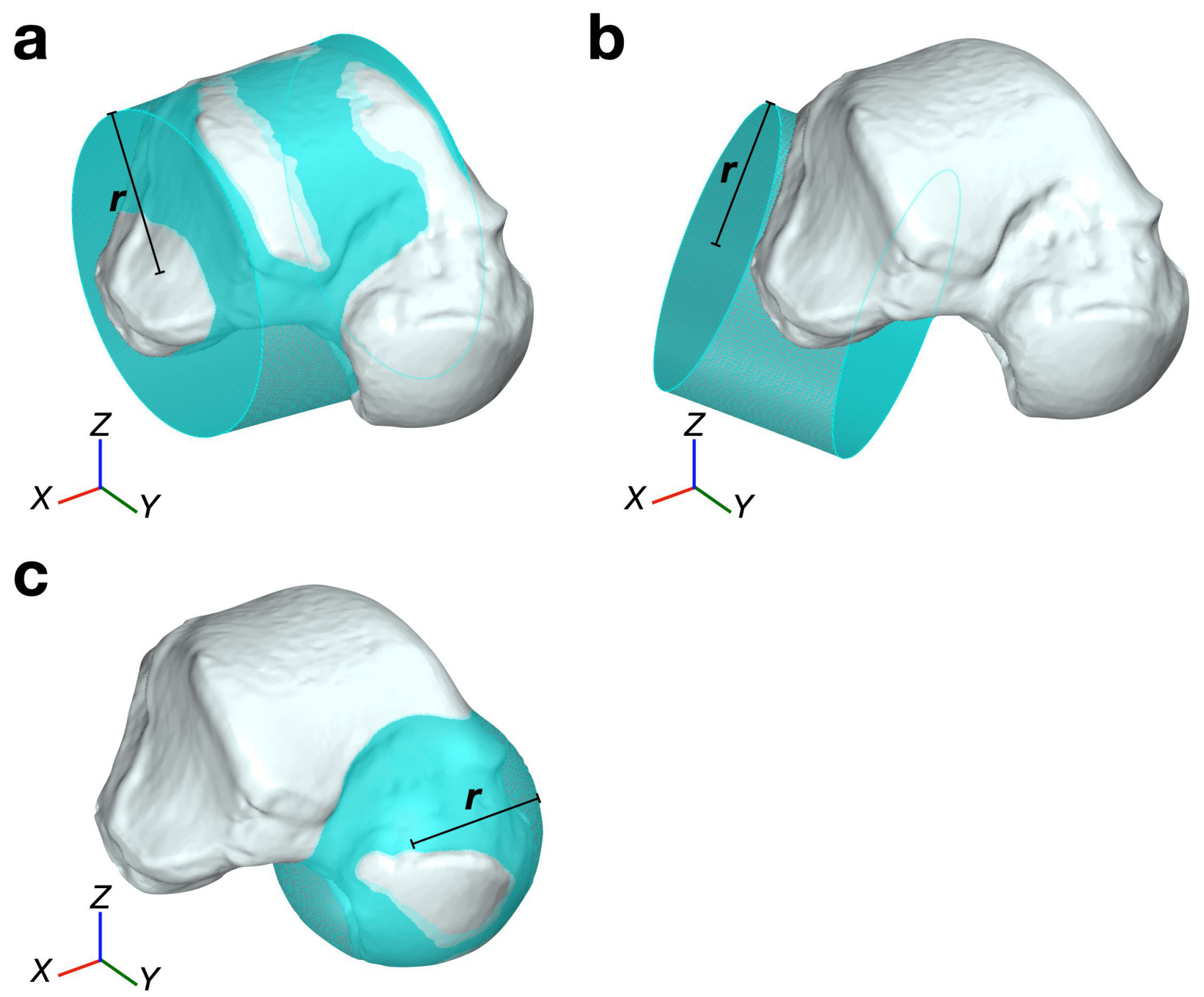

\section{Figure 3}

The curvatures of the articular surfaces of the talus. Cylinders and sphere are fitted to the superior trochlea (a), posterior calcaneal articular surface (b), and navicular articular surface (c). The radii (r) of the cylinders and sphere are calculated and normalized by the talar length, and the curvatures are determined. 
(a)

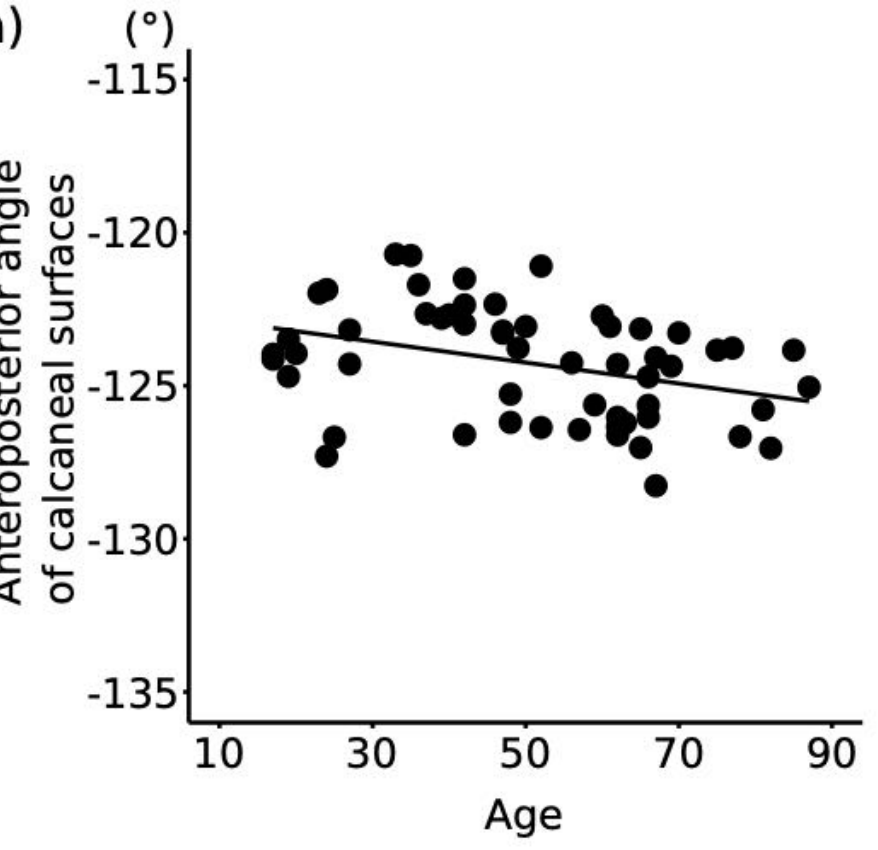

(b)

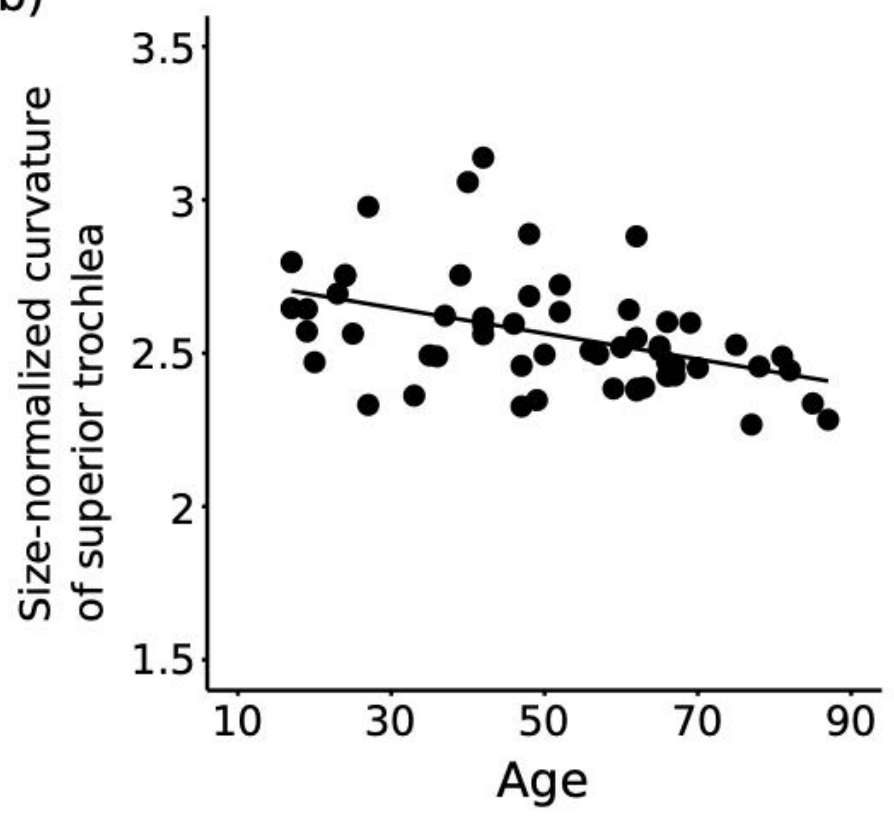

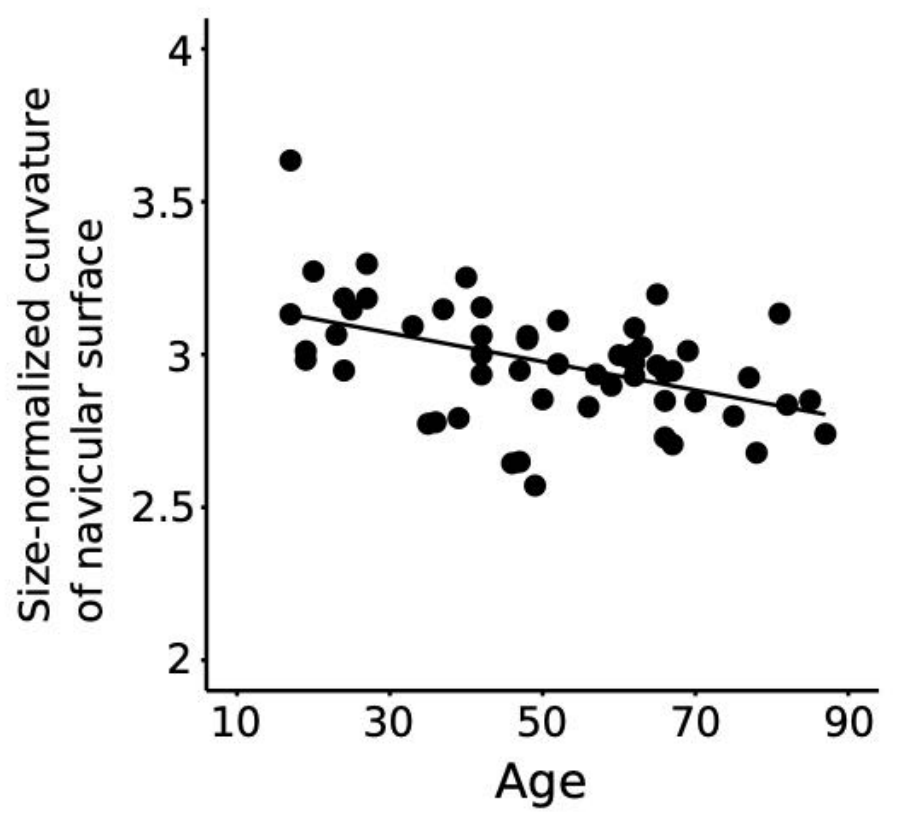

Figure 4

Bivariate plots of the superoinferior angle of the calcaneal articular surfaces (a) and size-normalized curvatures at the superior trochlea and navicular articular surface (b) relative to age. Age is significantly correlated with the superoinferior angle $(R=-0.357, P<0.01)$ and curvatures at the superior trochlea $(R=$ $-0.436, \mathrm{P}<0.001)$ and navicular articular surface $(R=-0.476, \mathrm{P}<0.001)$. 